\title{
Application of Cost Sensitive VPRS and Multi Fractal BP Neural Network in Construction of Intelligent Call Center System
}

\author{
Hongsheng $\mathrm{Xu}^{1}$, Ruiling Zhang ${ }^{1,2^{*}}$,Chunjie $\mathrm{Lin}^{1}$ and Youzhong $\mathrm{Ma}^{1}$ \\ ${ }^{1}$ Luoyang Normal University, Henan Luo Yang, 471022, China \\ ${ }^{2}$ School of Computer Science and Technology, Wuhan University of Technology, \\ Hubei Wuhan, 430070, China \\ *Corresponding author, E-mail: zhangruilingls@163.com
}

\begin{abstract}
Intelligent call center is also known as the customer service center or telephone service center, it is a kind of integrated information service system. This paper analyzes the classification method based on cost sensitive variable precision rough set. And in this paper, we use the attribute weighted cost sensitive rough set classification method based on established and customer level, customer history records, agents business related dynamic queuing strategy. In addition, this paper improves the multi fractal BP neural network algorithm for the call center customer classification. Improved algorithm is able to use multifractal fluctuation and BP neural network to predict the call center traffic and seat allocation. The paper presents construction of intelligent call center system based on cost sensitive variable precision rough set and multi fractal BP neural network. Experimental results show that novel method proposed can classify customers, reduce the impact of missing data and noise data, and improve the efficiency of customer satisfaction and intelligent call.
\end{abstract}

Keywords: Intelligent call center; Variable precision rough set; Cost sensitive; BP neural network; Multi fractal

\section{Introduction}

Call center is also known as the customer service center or telephone service center, it is a kind of integrated information service system based on CTI technology, make full use of communication network and computer network. Call center to use the traditional counter business telephone inquiry way instead of. Call center is able to provide service 24 hours a day, and has a better service than the counter service interface, users do not have to go to the office, as long as the phone can quickly get the information to solve the problem is convenient, fast, increase customer satisfaction for business services.

Early call center, the role of the advisory services is mainly. Start is to transfer some of the user's call to the answer desk or expert. To transfer the call and response with increased, began to build an interactive voice response (IVR) system, this system can be the most common question by the machine, automatic operator response and processing, the call center can be called the second generation call center.

There are three types of call center platform: one is based on the traditional switch mode (PBX mode); the two is based on the voice card mode; the three is the integrated platform based on the PCI Compact switching architecture. The last model uses advanced CTI technology, which can be used in a PCI Compact server on the integration of computer telephone integration (CTI), switch (PBX) and IP phone (VoIP), and so on more than 10 kinds of technology.

Compared with the qualitative analysis method, the quantitative analysis technique can deal with complex and massive problems, and the accuracy of the results is relatively high. The variable precision rough set model is Ziarko, a rough set model is proposed, and 
the model is set up by setting the precision standard parameters [1]. The variable precision rough set model is applied to the customer classification. The feature extraction can be effectively extracted from the noise and incomplete sample information. The system's anti noise capability can be improved and the complexity of the system can be reduced.

Modern call center, the application of computer telephone integration (CTI) technology is to make the call center service functions greatly enhanced. CTI technology is a medium of telephone voice; the user can press the button on the phone to operate the call center computer. Access to the call center can be a subscriber telephone dial-up access, fax access, computer and modem (MODEM) dial-up connection and Internet address (IP address) access, etc., users can receive a call center task prompt, in accordance with the voice of the call center, you can access to the database, access to the required information services. And storage, forwarding, query, exchange, etc. You can complete the transaction through the call center. Call center to use the traditional counter business telephone inquiry way instead of. Call center is able to provide service 24 hours a day, and has a better service than the counter service interface, users do not have to go to the office, as long as the phone can quickly get the information to solve the problem is convenient, fast, increase customer satisfaction for business services.

Artificial neural network, which is based on mathematics, neurology, physics, and computer and so on, is a network which is connected by a large number of processing units, which can be used to simulate the basic features of human brain. The combination of rough set attribute reduction and data mapping of neural network can improve the accuracy and efficiency of customer classification. In this paper, using attribute weighted cost sensitive classification method based on rough set, the establishment of dynamic related customer level, customer history, agent business volume queuing strategy to improve the quality of service.

In this paper, a (over Internet Protocol) technology is designed to realize the electronic commerce call center management system based on VOIP (Voice). This thesis combines theory with rough set BP neural network to classify the customers and agent queue based on traffic prediction, and optimization of call center scheduling based on chaos and fractal theory. The paper presents construction of intelligent call center system based on cost sensitive variable precision rough set and multi fractal BP neural network.

\section{Research on Decision Rough Set Analysis Based on Cost Sensitive}

In order to make the rough set has the fault tolerance and risk cost sensitivity, Yao et al proposed a new DTRS set (rough). In the definition of rough set approximate set, the model has the ability to deal with the data, and has the ability to deal with the data, and the rough set model with the risk sensitivity is established.

In the process of classification, the cost sensitive analysis mechanism is introduced, which is suitable for dealing with the problem of non balanced cost sensitive classification. Therefore, it is also called the cost sensitive decision rough set model.

Knowledge and classification is in rough set theory. Therefore, the rough set is based on the object in the domain, and the uncertainty is described by the upper and lower approximations of the set.

Definition 1: Given information system $S=(U, A)$, for any object subset $X \subseteq U$, and the relation to the upper approximation set $P \subseteq A$ and the lower approximation set defined as:

$$
\begin{gathered}
\bar{R}_{P}(X)=\bigcup\{Y \in U / I N D(P) \mid Y \cap X \neq \varnothing\} \\
\underline{R}_{P}(X)=\bigcup\{Y \in U / I N D(P) \mid Y \subseteq X\}
\end{gathered}
$$

Rough set theory can well reduce the data set, but it will be over reduced, resulting in the loss of the necessary information, so that the system's generalization ability. 
Knowledge reduction based on variable precision rough set theory can effectively prevent the loss of essential information, and increase the necessary redundancy by setting parameters, so that the system has good performance in generalization ability, as is equation(3), fault tolerance and noise suppression. Attribute subset is defined as the positive region of decision attributes.

$$
\beta^{1}(t)=\sum_{i=i_{1}}^{i_{2}} b_{i}^{1}(t)
$$

The aim of value reduction is to extract decision rules, so these missing attribute values are sure to be reduced, and attribute reduction is different, value reduction is for each object. Although there are no redundant attributes for the whole decision table, there are still some properties for each object, which can be removed by removing these attributes. According to the definition of the general value reduction algorithm, the basic description is as follows: for each rule in the rule set, the arbitrary conditional attribute, if you remove the attribute, and other rule conflicts in the collection, you can remove the condition attribute from the rule.

Definition 2: given information system, for the set of attributes, and $P, Q \subseteq A$, and $\forall G \subset P, P O S_{G}(Q)=P O S_{P}(Q), \forall B \subset G$, that is $P O S_{B}(Q) \neq P O S_{G}(Q)$, it is established that the relative reduction of the conditions relative to the conditions, the intersection of all relative reduction is referred to as the relative core..

$$
B N R_{P}^{\beta}(X)=\{x \in U \mid \beta<C(E, X)<1-\beta\}
$$

Definition 3: Set to an object's state collection $\Omega=\left(\omega_{1}, \omega_{2}, \ldots, \omega_{s}\right)$, a set of possible decisions for the object $P\left(\omega_{j} \mid x\right)$ [2]. The condition that the object has a state conditional probability, which $\lambda\left(a_{i} \mid \omega_{j}\right)$ is expressed in the state to take the decision of the price. For the object is to take the expected risk of decision-making.

$$
R\left(a_{i} \mid x\right)=\sum_{j=1}^{s} \lambda\left(a_{i} \mid w_{j}\right) P\left(\omega_{j} \mid x\right)
$$

The algorithm of rough set theory is studied. The algorithm of attribute reduction and rule extraction are studied. For example, the combination of attribute importance, information measure based heuristic algorithm, and other intelligence algorithms. For example, the combination of neural network and rough set theory is used to improve the convergence speed of neural network, and support vector machine SVM.

The ultimate goal of knowledge reduction is not only to reduce the conditional attribute, but also to compress the data quantity, but to lay a good foundation for the knowledge discovery. The task of knowledge discovery is to find out the inner relationship between knowledge and the decision rules. Rule extraction is actually a reduction of decision rules, which is mainly about the value of conditional attributes. In the decision table, each row corresponds to a decision rule. The core value of the conditional attribute of the decision rule is calculated, and the condition attribute value of the decision rule is obtained. If a repeat line appears in the reduction table, it is deleted because it represents the same decision rule.

Approximate accuracy reflects the accuracy of the classification of the attributes, and for a property, it is the ratio of the number of samples in the positive region and the total of the positive region. The correlation degree (also known dependence) of the conditional attribute subset and the decision attribute is defined as the following formula (6).

$$
R_{i}=R_{i-1} \cup\left\{c_{j}\right\} \rightarrow \min \left(\Delta \gamma_{P \cup C}^{\beta}\left(R_{i-1} \cup\left\{c_{j}\right\}, D\right)\right), c_{j} \in C-R_{i-1}
$$


Theorem 1: The critical point $\forall \alpha \in \bar{B}_{P}^{\beta}$ that can change the distribution of the upper and the distribution point set, $\alpha_{i}$ and $\alpha_{i+1}$ are the upper $\bar{B}_{P}^{\beta}$ and the middle of the distribution $\left(\alpha_{i}, \alpha_{i+1}\right)$.

From the upper and lower distribution of discernibility matrix definition can be seen, for any property in a single conjunctive form is variable precision rough set kernel functions can be seen from the distinction between condition attributes and decision attribute reduction $\mathrm{D}$ is all its minimal disjunctive conjunction in $\mathrm{C}$.

Theorem 2: The critical point $\forall \alpha \in \underline{B}_{P}^{\beta}$ of the potential change of the distribution of the lower distribution, and the distribution of elements in the set of the lower distribution point set, $\alpha_{i}$ and $\alpha_{i+1}$ and the two adjacent critical points in the interval, then the distribution of the lower distribution $\underline{B}_{P}^{\beta}$ in the interval $\left(\alpha_{i}, \alpha_{i+1}\right)$.

It is proved that under the $\beta$ upper (lower) distribution of the two critical points, the distribution of the critical point set is the critical point, which can be divided into a finite number of stable ranges, and the infinite values are converted to a finite value $\beta$. Users can accord need, such as approximate accuracy, $\beta$ classification quality and so on, a clear choice.

In incomplete decision table, the object in the domain is divided into a series of compatible particles, which is covered by the general system [3]. Therefore, it is easy to prove that the compatibility between the two objects satisfies $\forall x, y \in U$ the following properties:

(1) if $(x, y) \in \operatorname{SIM}(P), S_{P}(x)=S_{P}(y)$ it is not established;

(2) if $(x, y) \notin \operatorname{SIM}(P), q_{i i}=\lim _{h \rightarrow 0^{+}} \frac{p_{i i}(h)-1}{h}=-\left(\lambda_{i}+u_{i}\right)$ it is not established.

In the decision rough set theory, the approximate set is expressed with other probability rough set theory. The threshold value of the decision making method is obtained by calculating the minimum risk (cost) of the decision.

The following properties show that, although the two objects are not distinguishable from the compatibility relation, the two objects are not distinguished. That is to say, the two is the same decision class. However, they may contain objects that are not the decision class. Therefore, it can't be used to distinguish the two objects from the same decision class with these two objects.

Theorem 3: $\forall x, y \in U$, if $G_{A}^{\beta}(x) \neq G_{A}^{\beta}(y), S_{p}(x) \cap\left(S_{A}(y)-S_{A}(x)\right)=\varnothing$, if $G_{A}^{\beta}(x)=G_{A}^{\beta}(y), \quad$ When $S_{A}(x) \neq S_{p}(x), \quad \forall x^{\prime} \in S_{p}(x), \quad G_{A}^{\beta}\left(x^{\prime}\right)=G_{A}^{\beta}(x)$, when $S_{A}(y) \neq S_{p}(y), \quad \forall y^{\prime} \in S_{p}(y), \quad G_{A}^{\beta}\left(y^{\prime}\right)=G_{A}^{\beta}(y) \Rightarrow P$ is $\beta$ there is a set of distributed coordination.

Definition 4 [4]: The improved upper and lower distribution $S=(U, C \cup D)$ of the upper and lower distribution can be defined in the decision table:

$$
Q=\left[\begin{array}{lllll}
-\lambda_{0} & \lambda_{0} & 0 & 0 & \ldots \\
\mu_{1} & -\left(\mu_{1}+\lambda_{1}\right) & \lambda_{1} & 0 & \ldots \\
0 & \mu_{2} & -\left(\mu_{2}+\lambda_{2}\right) & \lambda_{2} & \ldots \\
0 & 0 & \mu_{3} & -\left(\mu_{3}+\lambda_{3}\right) & \lambda_{3} \\
\vdots & \vdots & \vdots & \vdots & \vdots
\end{array}\right] .
$$


In order to simplify the problem, consider a two value decision problem. For the property itself, the state set is $\Omega=\left(C, C^{C}\right)$, the decision set is $A=\left(a_{P}, a_{N}, a_{B}\right)$, for the loss function, $\lambda_{P P}, \lambda_{N P}$ and $\lambda_{B P}$ are the object belongs to the collection when the action is taken $a_{P}, a_{N}$ and $a_{B}$, and the loss is represented by an object that does not belong to the collection, and the loss.

Relative reduction reflects the relationship between the classification and the other classification, and the relative reduction does not change the ability of the object from one classification to another. Similarly, the relative core is the only one, and the relative reduction is the basis of the decision table attribute reduction.

Rough set the basic idea is to use defined sets to describe can define a collection, and proposes a concept of upper approximation and the lower approximation sets are defined. In the classical rough set approximation is based on the concept between qualitative relations (i.e., contains or disjoint empty) defined and does not take into account the concept intersection degree, so it is not available to many practical problems. In order to solve the rough set model is too strict, lack of fault tolerance; people put forward the probability of various type extension of rough set model.

Definition 5: if $S=(U, C \cup D) \quad, \quad U / I N D(A)=\left\{C_{1}, C_{2}, \ldots, C_{m}\right\}$,so $G_{C}^{\beta}(x)=\left\{D_{j} \mid \mathrm{x} \in \bar{R}_{P}^{\beta}\left(D_{j}\right)\right\}, M_{C}^{\beta}(x)=\left\{D_{j} \mid \mathrm{x} \in \underline{R}_{P}^{\beta}\left(D_{j}\right)\right\}$,

$$
D_{1}^{* \beta}=\left\{\left\{C_{i}, C_{j}\right\} \mid M_{A}^{\beta}(x) \neq M_{A}^{\beta}(y)\right\}, D_{2}^{* \beta}=\left\{\left\{C_{i}, C_{j}\right\} \mid G_{A}^{\beta}(x) \neq G_{A}^{\beta}(y)\right\} .
$$

The total price of the sample in the attribute subset is the sum of the false classification and the test cost.

After the information table of attribute reduction and value reduction, we can get the rules, and use a reduction set $\mathrm{S}=(\mathrm{U}, \mathrm{A})$ to produce the rules of the process is quite direct. Intuitively, each reduction is used to form a decision rule for each object in the decision table, as long as the proper attribute values are read from the table. In the form of a similar logic language, a decision rule is expressed as a decision rule, and the alpha and beta are called a decision rule.

In the process of data mining, rough set theory is the core of knowledge reduction and rule extraction analysis. The algorithm is based on knowledge reduction, and the decision or classification rules are derived through knowledge reduction. The knowledge processing model: the initial data of the initial data is not available for the matrix reduction set mining rules.

In rough set theory, knowledge is considered as classification ability. People's behavior is based on the ability to distinguish real or abstract objects. The so-called knowledge, that is, on the subset of the domain $\mathrm{u}$ for $\mathrm{u}$ on the concept, and agreed to the empty set is also a concept, the concept of the family of sets called u knowledge; the knowledge base of knowledge is the knowledge base of $U$. The non empty finite set of the $U$ object is discussed [5]. The so-called basic knowledge, that is, on the domain $\mathrm{u}$, the equivalence relation family $\mathrm{R}, \mathrm{P} \mathrm{R}$ and $\mathrm{P}=$, is not can distinguish between all the equivalence class of the collection, the quotient set. The basic concept of knowledge $\mathrm{P}$ is called the equivalence class.

Definition 6: $S=(U, A)$ is not complete information system, The calculation of reduction can generally be used as a starting point, and the calculation of the core can be obtained by definition, and can be obtained by the identification matrix is defined by the determination of the compatibility relation:

$$
\lambda p_{n}=n \mu p_{n+1}, p_{n+1}=\frac{\lambda}{n \mu} p_{n}=\frac{\rho^{n+1}}{n \cdot n !} p_{0}
$$

In this application, the customer registration information of a certain enterprise is collected based on rough set data mining, and the conditional attribute and decision 
attribute of the decision attribute are obtained by the initial processing, and the number of attributes is obtained. Because of the use of set Rough theory to deal with the decision table, the decision table of the value of the discrete value, after discrete treatment, such as Table 1 shows the decision table. $\mathrm{S}=<\mathrm{u}, \mathrm{R}, \mathrm{V}, \mathrm{f}>$, the design of domain for samples, which $R=C$-shaped $D, U=\{1,2, . .9\}$, condition attributes $C=\{$ age, gender, marital status, education, income $\}$ and decision attribute $\mathrm{D}=\{$ whether to buy $\}$. Before using the corresponding algorithm is for attribute reduction.

In the process of classification of the decision table, there may exist much reduction. If the intersection of these sets is the core, it is the basis of computing all the reduction, is the most important part of the collection, the knowledge can not delete it. The core attribute is a key attribute of classification, so how to calculate the properties of the kernel in the information system? The usual method is to delete the redundant instances and the non associated attributes, and then remove the redundant attributes of each instance, and then calculate the minimum reduction, as is shown by equation(9).

$$
F\left(x, y, \frac{d y}{d x}, \cdots, \frac{d^{n} y}{d x^{n}}\right)=0
$$

The rules expressed in the positive region are determined by the rules of $\mathrm{X}$. The rules that are derived from the negative region are not the rules of $X$, and the rules derived from the boundary regions are likely to be X, which reflects the basic idea of "three decisions". However, the LAK Paw rough set does not take into account the fault tolerance of the rule, which requires the introduction of conditional probability, probability rough set and other related concepts.

Decision model of rough set theory in rough set approximation sets are defined, with fault tolerance probability of inclusion relation instead of strictly algebraic inclusion relation and the data processing with fault tolerance, and the Bayes risk decision-making method to determine the concept of decision boundary, established the risk cost sensitive characteristics of rough set model, provides effective solutions for people from the data acquisition cost sensitive risk characteristics of decision knowledge.

Theorem 4: In the incomplete decision table $S=(U, C \cup D)$, the minimal disjunctive normal form $\quad F_{l}^{\beta}=\bigvee_{k=1}^{t}\left(\bigwedge_{s=1}^{q_{k}} a_{i_{s}}\right) \quad, \quad$ respectively $u=\frac{x^{2}}{2} y+\ln |y|$, $\left\{T_{l k}: k=1,2, \ldots, t\right\}(l=1,2)$ is the set of all upper and lower distribution reduction.

According to the cost matrix and the minimum risk Bayes decision method, the three kinds of $\mathrm{aP}, \mathrm{aN}$ and $\mathrm{aB}$ are calculated respectively. In rough set theory, because objects $[\mathrm{x}] \mathrm{R}$ equivalence class of $\mathrm{X}$ has exactly the same feature description, where you can with equivalence classes $[\mathrm{x}] \mathrm{R}$ as characteristic $\mathrm{x}$ description form in general, sample classification results with the test attribute set is closely related. With the increase of test attributes, the classification accuracy of the sample is higher, the less the result is, the smaller the error is. Therefore, the test attribute set containing more attributes usually has a small error classification cost. But in the actual classification problem, the attribute value of the sample has a certain price, which is the test cost.

In this paper, the optimal attribute set can be reduced to a large extent. The reason is that the optimal attribute set contains only a few of the attributes with high classification performance, which has a great contribution to reduce the total cost of the classification. Although the reduction of the number of attributes can cause the increase of the error classification cost, it also reduces the test cost of the classification, so that the optimal attribute set has a lower total cost in the classification.

\section{The Combination of BP Neural Network and Multi Fractal Theory}


Neural network in the practical application of input samples can be divided into three categories: training samples, test samples and test samples, the corresponding stage for: training, testing and validation phases, in reality tend to test and verification phases merged into a stage, of which the first stage is to adjust the network weights to show the problem domain, in the network learning can enter the second and the third stage, weight is fixed, the actual test data is input to the neural network, the validation check network classification ability.

Fractal is a powerful mathematical tool for studying and dealing with irregular graphics, which provides new ideas and methods for dealing with nonlinear systems. People call it and the dissipative structure and chaos theory are three important discoveries in the middle of 1970 s.

BP neural network sample dependence problem: the approximation and generalization ability of the network model is closely related to the typical characteristics of the learning sample. Back propagation is to calculate the error signal (the difference between sample output and network output) by the method of gradient descent. The following is the weights of each layer specific process (i.e. BP learning algorithm XI): definition of network output error $\mathrm{E}=1 \mathrm{~L}(\mathrm{D} / \mathrm{O}) 2=\operatorname{sigma}(\mathrm{d} \mathrm{k}-\mathrm{OK}) 2$, which in turn to hidden layer $2 \mathrm{~K}=1$.

BP network learning algorithm, the weight of each layer is adjusted by the learning rate, the error value of the layer and the input vector of the layer, the error signal of each layer is related to the error signal in front of each layer, as is shown by equation (10).

$$
\square w_{j k}=\eta\left(d_{k}-o_{k}\right) o_{k}\left(1-o_{k}\right) y_{j}
$$

Neural network is a collection of individual parallel processing elements; we have been inspired by biological neural system [6]. In nature, the network function is determined by the ganglia, we can do the specific work by changing the weights of the connecting points. A general neural network can be regulated, or be trained, such that a particular input can be obtained by the required output. As shown below. Here, the network is adjusted according to the comparison of the output and the target until the network output and the target matching. As a typical example, many of the input / target methods have been used to train the neural network in the supervised model. Neural networks have been used in various fields to achieve a variety of complex functions. These areas include: pattern recognition, identification, classification, voice, translation and control system.

For all training (sampling) input, the weights are changed, and the weights are corrected. The weights of BP neural network are directly proportional to the output error, and the output of the training target can only approximate 1 and two 0 , but not 1 and 0 . Dimensional, when using 1 as the target value for training, all output actually showing a value higher than 0.9 ; when we use 0 as the target value for training, all output actually show a value that is less than 0.1 ; this performance is considered to be satisfactory.

The three layer BP neural network structure, the input vector is a threshold for the hidden layer nodes, and the output vector of the hidden layer is the output vector of the output layer. The input layer to the hidden layer between the weight matrix, the column vector representation of the first hidden layer node corresponding to the weight vector, hidden layer to the output layer between the weight matrix, as is shown by equation(11).

$$
y_{i}=f\left(\sum_{i=0}^{n} v_{i j} x_{i}\right) \quad j=1,2, \ldots, m
$$

The functional characteristics of the artificial neural network are determined by the topology structure and the intensity of the synaptic connection. The neural network can change the connection weights and the topology of the network, so as to make the actual output of the network continuously close to the desired output.

The structure and structure of BP network is the same as that of the feed forward network. The following points are discussed here. The transfer function of the BP 
network, which is commonly used in 1, is logsig, Tansig, sometimes used in linear function purelin. When the last layer of the network uses a curve function, the output is limited to a very small range, and if the linear function is used, the output can be arbitrary. These three functions are the most commonly used functions in the BP network, but you can also create other differentiable transfer functions if needed.

We use BP to create the newff neural network; it defaults to use initnw to initialize the first layer. If we want to Rands re initialization BP of the first layer weights and bias, we use the following command: = net.layers $\{1\}$.initFcn'initwb'; = net.inputWeights $\{1,1\}$.initFcn'rands'; $=$ net.biases $\{1,1\}$.initFcn'rands'; $=$ net.biases $\{2,1\}$.initFcn'rands'; net $=$ init (net); network simulation (SIM) network simulation function SIM simulation of a network.

BP neural network model is including the input and output model, function model, error calculation model and self-learning model. (1) a node output model of hidden nodes output model: $\mathrm{Oj}=\mathrm{f}$ ( $\operatorname{sigma} \mathrm{Wij} * \mathrm{Xi}-\mathrm{q}$ J) (1) output node output model: $\mathrm{Yk}=\mathrm{f}$ (sigma Tjk * Oj-q K) (2) F- non linear function; Q neural unit threshold.

Although there are many researches on the fuzzy neural network at home and abroad, but most of the fuzzy neural network is focused on the BP neural network and fuzzy logic. Because of the inherent defects of BP network, there are some defects in the network. These defects mainly include: the slow convergence speed, easy to fall into local minimum, real-time learning ability and generalization ability to be enhanced. Therefore, it is urgent to develop new fuzzy neural network and explore its application in the industry process.

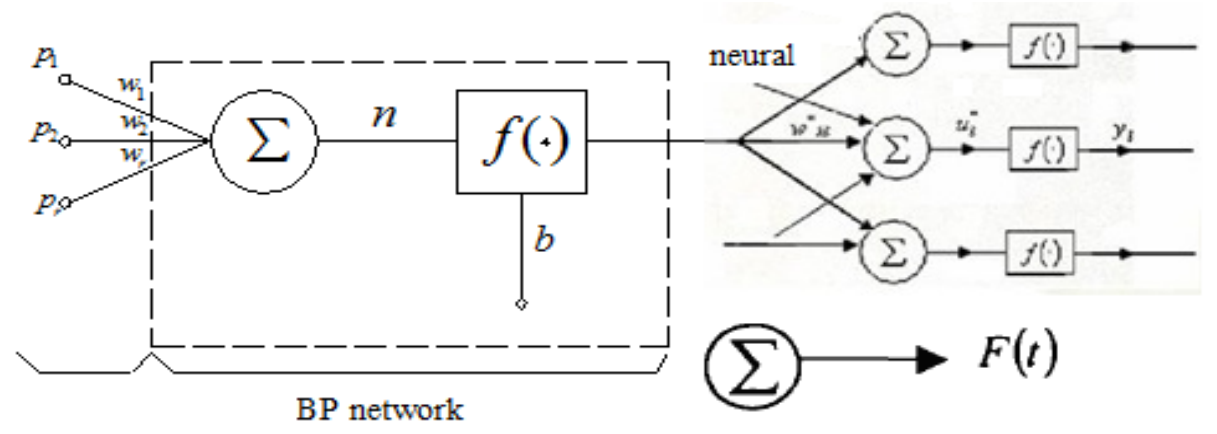

Figure 1. The BP Neural Network Model Diagram

Figure 1 is a neural network structure, in which the hidden layer and output layer weights, $\mathrm{X} 2, \mathrm{X} 1$ is the input value of neural network, $\mathrm{y}$ is the output value of the network, YP is a teacher signal, e neural network for the actual output and the expected output error. In this neural network, node 1, 2 is the input layer, node 3, 4, 5 is the hidden layer, node 6 is the output layer; input layer and hidden layer between the weights in order to w13, 14, w15, w23, W24, W25, hidden layer and output layer between the weights for W36, w46, w56, subscript node numbering; hidden layer and output layer node threshold in turn is from 3,4,5; $\mathrm{K}$ from 3,4,5) as input layer and the hidden layer weights.

The mathematical demonstration of the back propagation characteristic is based on the following two concepts: let y be a smooth function of some variable xi. We want to know how to achieve the incremental changes of the initial value of each $\mathrm{Xi}$, so as to increase the $\mathrm{y}$ value as fast as possible, the initial value of each $\mathrm{Xi}$ should be proportional to the partial derivative of Xi with $\mathrm{y}$.

When the input signal is disturbed, the output will not have a large distortion. The information distribution of the neural network, but also to train the neural network has a strong association. 3) Parallelism. Neural network is a simulation of the structure and function of the human brain, but it is more emphasis on the simulation of the structure. Each neuron is independent of processing information, and they receive input and output. 
Such parallel computing makes it possible to use the information in the proper time, which is the basis of the next generation of intelligent computer.

The output value of each neuron is close to zero, so that the weight value of each neuron can be ensured to be adjusted to the maximum of the $\mathrm{S}$ type activation function. So, the random number is between the initial weights and the initial weights in $(-1,1)$. The learning rate of determines the weight of each cycle. The large learning rate can lead to instability of the system, but the small learning rate can lead to a long training time, which may be slow, but can guarantee that the error value of the network does not jump out of the surface of the error, and eventually tends to be the smallest error margin [7]. So in general, it tends to select a smaller learning rate to guarantee the stability of the system, as is shown by equation (12).

$$
x_{1}^{1}(t+1)=\beta^{1}(t) \sum_{i=i_{1}}^{i_{2}} b_{i}^{1}(t)^{*} x_{i}^{1}(t)-s_{0} \alpha N^{1}(t)
$$

Definition 7: Assumed to be nonempty sets in Euclidean space, the diameter is defined as $|U|=\sup \{|x-y|: x, y \in U\}$. If it is a set of numbers that are not more than a set of numbers or a finite number of diameters not exceeding the set of sets: $F \subset \cup_{i=1}^{\infty} U_{i}$, for any $0 \leq\left|U_{i}\right| \leq \delta$, there are, then, a cover that is called $\left\{U_{i}\right\}$.

BP neural network object and initialize the network weight and bias, so the network can be trained. We may have to re initialize the weights or for a custom initialization. Here is a detailed procedure for the initialization. Before training a feed forward network, the weights and bias must be initialized. Initialization weights and bias of the work is with the command init to achieve. The function receives a network object and is initialized with the weights and returns to the network object.

BP neural network has been successfully used in the field of function approximation, pattern classification, system modeling, pattern recognition, signal processing, etc.. BP has the advantages of simple network structure, strong nonlinear approximation ability, fast convergence speed and global convergence. RBF has many aspects to be further studied, especially how to select the number of radial basis functions, as is shown by equation (13).

$$
f^{\prime}(\xi)=\frac{f(M)-f(x)}{M-x}
$$

Definition 8: $F \subset R^{n}$ set, where the study of the fractal body. Remember that the smallest number of dimensions that $H_{\delta}(F)$ can be covered by a variable length is defined as the number of dimensions of the box [8].

$$
D_{B}=\lim _{\delta \rightarrow 0} \frac{\log N_{\varsigma}(F)}{-\log \varsigma}
$$

Assuming that there are two inputs to 1 value, and while the rest are 0 values. $\mathrm{H} 1$ and $\mathrm{H} 2$ as the hidden nodes, and has the function of. The goal of the BP neural network is to determine whether the input of the two individuals met. The task of the network is to learn any one of the 3 people in the top group is an acquaintance of any of the 3 people in the following. If the output of the network is greater than 0.9 , then it is judged that the two are met; if the output is less than 0.1, the judgment is not known; other results are considered as fuzzy

There are many variables in the back propagation algorithm, which will be discussed in this chapter. The simplest way to use the back propagation learning algorithm is to update the weights and bias of the negative direction of the gradient. The recursive algorithm can 
be written as: $\mathrm{xk}-=\mathrm{a} \mathrm{k} \mathrm{g} \mathrm{K} \mathrm{xk+1} \mathrm{where} \mathrm{XK}$ is the current weight and bias vector, $\mathrm{kg}$ is the current gradient; $\mathrm{K} \mathrm{A}$ is the learning rate.

Determine the function, usually choose a non - linear S-type function (15) establish knowledge base of safety evaluation system by BP neural network learning confirmation network structure includes: input, output and the number of hidden nodes and to reflect the combination of degree of network weights during; that is, with the reasoning mechanism of the safety evaluation of the system knowledge base and are evaluated. (6) The safety assessment of the actual system is trained by the neural network to evaluate the characteristics of the system, and the input to the neural network which has been trained.

$$
f(b)-f(a)=\frac{f^{\prime}\left(x_{3}\right)}{\frac{1}{x_{3}}}(\ln b-\ln a)=x_{3} f^{\prime}\left(x_{3}\right) \ln \frac{b}{a}
$$

Where there is a power law relation $(X, \mu)$ between the unit measure and the power law, the Holder exponent is called $\mu_{\alpha} \sim \delta^{\alpha}$. Since it controls the singularity of the probability density, it is also called the singularity exponent.

BP neural network and Boltzmann are all of this type. In the forward network without feedback, the signal is passed through a neuron, and the processing of the neuron is over. In the network, the signal is transmitted repeatedly between neurons, and the network is in a state of constant change. Signal from an initial state, after a number of changes, will achieve a balanced state. According to the structure of the network and the characteristics of neurons, the operation of the network is also possible to enter the periodic oscillation or other such as chaotic equilibrium state.

\section{Design of Intelligent Call Center System Based on Cost Sensitive Variable Precision Rough Set and Multi Fractal BP Neural Network}

Call center is a number of companies for the user service and the establishment of the company. In the early 1980s, Europe and the United States telecommunications companies, airlines, commercial banks and other to keep close contact with the user, the support of computer application, using telephone as and user interaction with the media, set up a call center (call center), also known as a call center, in fact, for the customer service center, so call center in foreign countries has been widely used [9]. Domestic situation is just the opposite. Since we began to introduce the concept of call center in the late ninety's, is now in the market cultivation and starting period, and the industry has begun to build the call center, so call center industry in China's annual growth rate of about 30\%. Due to the huge market demand in China, when the world call center market tends to saturation, some companies with advanced technology, equipment and management experience will turn to china.

The call center system will voice query, self-service, artificial service closely integrated, the shopping website sales, distribution of resources, supply chain resources, customer resources, such as through the network integration, establish a rapid response mechanism of online shopping, enhance customer groups and shopping website interaction, and utilization of resources to achieve the uniform distribution, in order to achieve maximum profit for the enterprise.

Generally speaking, the system is mainly determined by the operating system of the core exchange, and then the design of hardware and software is reasonable and the degree of product, as well as the function of the hardware and the design of the integration of the system has a great impact on the stability of the system. Generally speaking, in the call center industry standards are not unified, multi vendor product integration will certainly reduce the stability of the system, and the system's recovery is a very complex process. 
The application of rough set combined with artificial neural network training sample set simplification, the artificial neural network with parallel processing, high fault tolerance and strong generalization ability, suitable for application in prediction, complex object modeling and control occasions. But when the neural network size larger sample is large, training time is too long, the inherent shortcomings is control neural network for the further practical is one of the main factors. Although all improve the training speed of the algorithm will continue to appear, problem is far from solved thoroughly. Of Jane training sample set, eliminate redundant data is another provided high training speed.

The classical rough set is based on equivalence relation, but in incomplete information systems, the equivalence relation is no longer applicable. Therefore, some scholars put forward a variety of extensions, which are able to retain the knowledge of the maximum retention system, and the relationship between the most current information systems that meet the relation formula is as follows.

$$
p_{0}=\left[\left(1+\rho+\frac{\rho^{2}}{2 !}+\cdots+\frac{\rho^{n}}{n !}\right)+\frac{\rho^{n+1}}{n !(n-\rho)}\right]^{-1}, p_{i, j}(t)
$$

Definition 9: In the given decision table $S=(U, C \cup D)$,

$\eta_{P}^{\beta}=\left(\bar{R}_{P}^{\beta}\left(D_{1}\right), \bar{R}_{P}^{\beta}\left(D_{2}\right), \ldots, \bar{R}_{P}^{\beta}\left(D_{n}\right)\right), \xi_{P}^{\beta}=\left(\underline{R}_{P}^{\beta}\left(D_{1}\right), \underline{R}_{P}^{\beta}\left(D_{2}\right), \ldots, \underline{R}_{P}^{\beta}\left(D_{n}\right)\right)$ if $\eta_{P}^{\beta}=\eta_{C}^{\beta}$, then is called distribution consistent set, if distribution consistent set, and any proper subset are not distributed coordination set, called on distribution reduction. (2) if $\xi_{P}^{\beta}=\xi_{C}^{\beta}$ subset set are not distributed coordination is called for lower distribution reduction.

It is found that the compatible particle is a set of objects with compatible features, and the object's compatibility is the collection of objects that are similar to the object.

The variable precision rough set model is an extension of the traditional rough set model. It can relax the definition of the approximate boundary of the standard rough set theory by setting the variable precision parameter $\beta$. Therefore, it will have a greater impact on the reduction results. In most cases, the selection of the value of the knowledge and experience of domain experts, therefore, how to determine the range of the value $\beta$ of the variable precision rough set theory has important significance. Some scholars have found that in the complete information system, the value of the number of fixed interval, the upper and lower distribution reduction results are stable.

Theorem 5: $\forall x, y \in U$, if $M_{A}^{\beta}(x) \neq M_{A}^{\beta}(y)$, then $S_{p}(x) \bigcap\left(S_{A}(y)-S_{A}(x)\right)=$ $\varnothing$; if $M_{A}^{\beta}(x)=M_{A}^{\beta}(y)$, when $S_{A}(x) \neq S_{p}(x), \forall x^{\prime} \in S_{p}(x), M_{A}^{\beta}\left(x^{\prime}\right)=M_{A}^{\beta}(x)$, $M_{A}^{\beta}\left(y^{\prime}\right)=M_{A}^{\beta}(y) \Rightarrow P, \mathrm{P}$ is a distributed coordination set.

Rough set is an effective method of data mining in database. We only need to input the database into rough set operation system, without any prior knowledge, the rough set algorithm can automatically learn the knowledge, which is the source of its wide application. And in the set theory of fuzzy sets, extension set, we also give the membership functions in advance.

In fact, it is a two-dimensional table, each table shows an object, and each column represents each attribute of the object, while the attribute is divided into two categories, the attribute and decision attribute [10]. As a result of an equivalence relation, a table can be expressed as a family of equivalence relations, namely knowledge base, so the knowledge reduction can be transformed to attribute reduction.

Algorithm 1: Variable Precision Rough Set and Distribution reduction algorithm based on Variable Precision Rough Set, Critical point set algorithm for computing parameters. 
Input: incomplete decision table $S=(U, C \cup D)$, Upper and lower distribution reduction critical point.

Output: the upper distribution reduction set and Lower distribution reduction set.

Step 1: calculate the compatibility class for all elements in the decision table;

Step 2: calculate all the compatibility classes of the decision table;

$$
\left\{S_{P}\left(x_{1}\right), S_{P}\left(x_{2}\right), \ldots, S_{P}\left(x_{|U|}\right)\right\} \text {; }
$$

Step 3: according to the definition of 9, the distribution of the identification matrix is calculated;

Step 4: calculate the compatibility class for all elements in the decision table;

Step 5: each object in the universe performs the calculation of the value of the object in the calculation;

Step 6: through the distribution of discernibility matrix are distributed on the resolution formula, and calculate the minimal disjunctive normal form;

Step 7:if $1>C \geq 0.5$, then $\bar{B}_{P}^{\beta} \leftarrow \bar{B}_{P}^{\beta} \cup C$,

$$
\frac{d^{2} y}{d t^{2}}+\frac{g}{l} \sin y=0
$$

Step 8: identification matrix distribution formula can be distributed through the resolution, and calculate the minimal disjunctive normal form;

Step 9: the end of the algorithm, the output reduction results;

Step 10: Sort the elements of the elements, and output.

In the integration of distributed IP call center model based on the business model and service process to meet the actual business process integration needs of the two development, and at any time according to business process changes to adjust. The call center system needs to meet the needs of the existing three CRM subsystem, the fund subsystem, the consumer credit subsystem and the settlement subsystem for the business process to meet the needs of the call user for a variety of business processing and business inquiries.

This system uses the service oriented architecture (SOA) business process integration method. Three CRM systems as a service provider, using WSDL (Web Services Description Language) to describe Web Services: and (Univemal Descrlption. On the discovery and integration) UDDI Registration Center released, registered Web Service: as the service requester through the UDDI Query Web service call center CRM system, finds the desired service can use soap (simple object access Protoc01) to bind and invoke these services, can not only ensure the original system data security and logical security, but also can realize the loose coupling between the system, so as to facilitate the restructuring and optimization of the process of the whole system.

In a large extent, rough set of positive region, negative region and boundary region provides the theoretical basis for the three-way decisions. Specifically, positive region corresponding to the rules, referred to as positive rules, said receiving; negative field corresponding to the rules, referred to as negative rules, rejected; rules corresponding to the domain boundaries, referred to as regular boundary, correspondence does not make a decision or postpone the decision. Whether accept or reject May with error, i.e., receive error or false rejection, which and statistics on a hypothetical received and rejected similar.

Obviously, attribute reduction is the minimal attribute subset of the system's classification ability, and the other kernel is the only one, but the reduction is not the only one. The kernel is contained in all reduction, and most of the heuristic attribute reduction methods are based on the calculation of the core is defined as: 


$$
\left\{\begin{array}{l}
x_{0}^{1}(t)=\left(1-c^{1}(t) f^{1}(t)\right. \\
x_{1}^{1}(t+1)=\beta^{1}(t) \sum_{i=i_{1}}^{i_{2}} b_{i}^{1}(t)^{*} x_{i}^{1}(t) \\
x_{i+1}^{1}(t+1)=\left(1-d_{i}^{1}(t)\right) x_{i}^{1}(t)
\end{array}\right.
$$

The main function of BP neural network is in the practical application of artificial neural network. Most of the neural network models are used BP neural network and its changes. It is also the core of the former network, which embodies the essence of artificial neural network.

For each sample of the two type of error function for the system to a training sample of the global error function for the error gradient descent method in order to modify the output layer weights of the modified formula for the weight of the modified formula [11].

Es for the control vector error and delay vector set, in which only consider the control rules for the use of error signal; TDL for time delay line; D as the output target; $y$ for the controlled output; $u$ is the control input; for CRS control rule set; HNO for BP neural network optimizer; HNE for BP neural network estimator; Phi output estimator is forecast model parameter identification for vector set.

Definition 10: Set is a bounded set $R^{n}$, we calculate the minimum number of balls $N(S)$

length $(\mathrm{D}=1)$, area $(\mathrm{D}=2)$ or volume $\mathrm{D}(=3)$, then $N(S) \sim S^{-D}$.

Algorithm 2: BP network training algorithm

Input: training sample set $P$

Output: weight $\mathrm{V}$ matrix of the network $\mathrm{W}$

Step 1: initialization weight matrix and sample count, number of training, error, initial training accuracy;

Step 2: assume the frequency parameter $\mathrm{k}=1$, draw the curve of the nonlinear function to be approximated;

Step 3: enter the first sample input to the network, calculate the output of each layer;

Step 4: initial weight and threshold training cycle count and save the output of each network layer and save the transmission error and save the weight and the threshold value $\mathrm{s}$

Step 5: calculate the actual output and the target output error;

Step 6: on each layer from $m$ to 2 reverse calculation (reverse); B. B. on the same node j g m, by 34.1 and 34.2) calculated delta JK;

Step 7: according to the error signal of each layer to modify the weight of each layer;

Step 8: Step 3: to select a set of input and target samples randomly from the learning sample of $M$, and return the step (3), until the global error of the network is less than a minimum value of $\mathrm{E}$, i.e., the network convergence. If the number of learning times is greater than the preset value, the network is unable to converge;

Step 9: if the algorithm ends; otherwise, the execution the end of the study.

BP network, it is a very important theorem, namely for any in the closed interval continuous function can be approximated by the single hidden layer BP network, and a three-layer BP neural network can complete mapping of arbitrary dimension $n$ to $m$ dimension. Therefore, this experiment use three layer BP network prediction, learning function: input layer to the hidden layer using function, hidden layer to the output layer uses logsig function. The first data in each set of data in the same condition as the test data is selected in the same condition, and the remaining data is the training data sample.

CTI technology is to telephone speech as a medium, when customers through telephone calls to the call center (call center) "can use the buttons on the phone to operate computer call center, will be able to receive the call center task tone, according to the call 
center voice prompt, can access database, access to the required information service, but also can realize the storage, transfer, inquiry, exchange, also can call the center through the completion of the transaction associated.

\section{Experiments and Analysis}

In this paper, using PHP technology to achieve a based on B / S mode based on electronic business of VoIP call center management system, the system mainly in the field of electronic commerce. The system includes call center based on platform module, the call center agent's platform function module, electronic commerce business management module, knowledge service module.

This paper uses rough set theory combined with BP neural network to queue the customer classification and reclined at the table, the chaos and fractal theory forecast traffic and optimization of call center scheduling, in order to improve the quality of customer service and reduce operating costs. System is mainly divided into call center platform, call center agents management platform, e-commerce business management platform and knowledge service management platform four internal subsystems, the system mainly completes the following functions.

(1) Seat call center management functions include: basic information management and account management module two. Basic information management: call history, until the dial, schedule management, bulletin management, scheduling management, queue management; account management: user management, role management, user identification, and user online statistics.

(2) E-commerce business management functions include: customer management and shopping management. Customer management: including customer information management, customer care, customer records, repair management, complaint management, questionnaire investigation, statistics analysis; shopping management: including commodity management and order management.

(3) Based on the analysis of multiple traffic volume time series analysis, using multifractal detruded fluctuation analysis algorithm from ordering the nonlinear feature extraction in agricultural products of traffic of historical time series and for call center traffic and seat allocation of prediction using the chaos and fractal theory.

(4) Knowledge service functions include: providing agricultural products knowledge service module, according to the business logic of the service classification, service to the user according to the intelligent classification module to respond.

Theorem 6: Incomplete decision table $S=(U, C \cup D)$, attribute subset $P \subseteq C$, $\forall x \in U, \quad \forall D \in U / I N D(D)$, the following conditions are established:

(1) if $C\left(S_{P}(x), D_{i}\right)<=0.5$, then $\mu(x, y)=e^{\int \phi(y) d y}$

(2) if $C\left(S_{P}(x), D_{i}\right)>0.5$, then $\mu(x)=e^{\int P(x) d x}=e^{\int-\frac{n}{x+1} d x}=(x+1)^{-n}$

In this paper, based on the variable precision rough set combined with BP neural network classification algorithm is the first according to the history of customer information, using variable precision rough set attribute reduction of the, simplify the customer information table, then the simplified data as training data for BP neural network, using the BP algorithm to train the network, BP neural network classifier, the new customer information using BP neural network classifier is classified to get customer category.

This paper based on attribute weighted price sensitive to rough set classification method, set up with the customer level, customer history records, agents business related dynamic queuing strategy, in order to improve the quality of service. Firstly, according to the experience of the expert to establish cost matrix and extracting test costs and misclassification cost minimum attribute set from the customer information table, based 
on the given minimum total cost classification rule set, according to the current user's incoming information, current idle agents information and rules set, queuing on the seats, making to get seats in the most suitable for the inbound customer service.

Algorithm 3: cost sensitive rough set classification rule generation and Multi fractal to trend Volatility Analysis algorithm

Input: decision table $S=(U, C \cup D)$, cost matrix $\mathrm{r}$, test cost function $\mathrm{T}(\mathrm{x})$.

Output: classification rule set $\mathrm{R}$

Step 1: calculate

$$
\lambda_{B}^{\beta}=\frac{\sum\left\{\left|{\overline{R_{B}}}^{\beta}\left(D_{j}\right)\right|: j \leq r\right\}}{|U|}
$$

Step 2: construct the mean value and sequence

$$
\vartheta^{\gamma}\left(X_{\alpha}, \eta_{\alpha}\right)=\lim _{\gamma \rightarrow \infty} \vartheta_{\delta}^{\lambda}\left(X_{\alpha}, \eta_{\alpha}\right)
$$

Step 3: calculate $\gamma^{\frac{1}{2}\left(\beta_{i, 1}^{C}+\beta_{i, 2}^{C}\right)}(C, D)$;

Step 4: A single hidden layer BP network is used to predict the input sample is 5 dimensional input vectors, because the input layer has 5 neurons, 20 neurons in the middle layer and the output layer of a neuron.

Step 5: perform the following operations as follows:

(1) For each of the attributes, the highest value of the total cost of the classification, so that $u=\frac{x^{2}}{2} y+\ln |y|$, Cost $_{=} \operatorname{Cost}_{a^{\prime}}^{\left.B \cup a^{\prime}\right\}}$;

(2) if $H_{B}^{\beta}=H_{C}^{\beta}$, then the execution step 4;

(3) if $N_{S}>v \geq 2 N_{s}$, then $F^{2}(v, s) \equiv \frac{1}{S} \sum_{i=1}^{s}\left\{Y\left[N-\left(v-N_{s}\right) s+i\right]-y_{v}(i)\right\}^{2}$;

Step 6: calculate the trend values for each fragment, fitting polynomial of the interval;

Step 7: calculate $\Delta f\left(\Delta f=f\left(\alpha_{\min }\right)-f\left(\alpha_{\max }\right)\right)$ the result of all minimum cost categories.

Step 8: the sequence of the sequence is decomposed into a non overlapping length

Step 9: $\alpha=h(q)+q h^{\prime}(q), f(\alpha)=q[\alpha-h(q)]+1$ according to the derived rule set.

Step 10: take the average of all the fragments to the fluctuation function

$$
\mathrm{f}(\mathrm{x})=\operatorname{Miscost}(x, B)+\operatorname{Testcost}(x, B), \quad r_{i j}=\frac{1}{6} \sum_{i=1}^{6} \xi_{i j}(k)
$$

Step 11: analysis and double logarithmic function to determine the scale of the volatility function, according to Legendre transform.

In the development process of this system adopts structured system development method, adopts the method of "from top to bottom and layer by layer decomposition" development strategy, through the structured design method, based on VoIP call center of e-commerce management system is divided into call center platform, call center agents management platform, e-commerce business management platform and knowledge service management platform four internal subsystem.

Finally, the results of the test for agricultural product electronic commerce call center business logic, the design and implementation of the based on VoIP intelligent call center management system, system in Apache environment with PHP language based, HTML and JavaScript language supplement the code, using MySQL as the number database system, to ensure the data processing, data access in the process of efficient, safe, the three layer architecture to manage code, to isolate the user layer and data layer, for the maintenance of project management and the late provides reliable guarantee. 
Full name of MVC is a model view controller, is the abbreviation of model (model View - controller (controller) and a software design paradigm for organization code separation method for a business logic and data display, the assumption of this method is if the business logic is gathered to a component inside, and user interface and around the interaction data can improve customization and personalization without the need to write business logic MVC is unique developed for traditional mapping of input, processing and output functions in the graphical user interface of a logical structure.

The call center platform mainly includes: the dial plan, pre dialing, dial results, speed dial, recording information, line information and call statistics, system parameter setting, data import, work setting, time setting, packet queue status, agent status monitoring function; seat call center management platform includes: call to record, dial, schedule management, bulletin management, scheduling management, queue management, user management, role management, user identification, user online statistics and other functions; business e-commerce management platform mainly includes: the function of customer information management, customer care, customer records, repair management, complaint management, questionnaire survey and statistics analysis including; knowledge service management: knowledge management, information retrieval and intelligent response.

Finally, the time complexity of the intelligent call system based on the cost sensitive variable precision rough set and multi fractal BP neural network algorithm is proposed. As shown in Figure 2. The experimental results show that the newly proposed method is better than BP neural network can provide more dynamic queuing strategy, customer call is assigned to appropriate agents, can effectively find, maintain and retain customers, providing customers with personalized service, improve customer satisfaction, optimize the call center of the allocation of human resources.

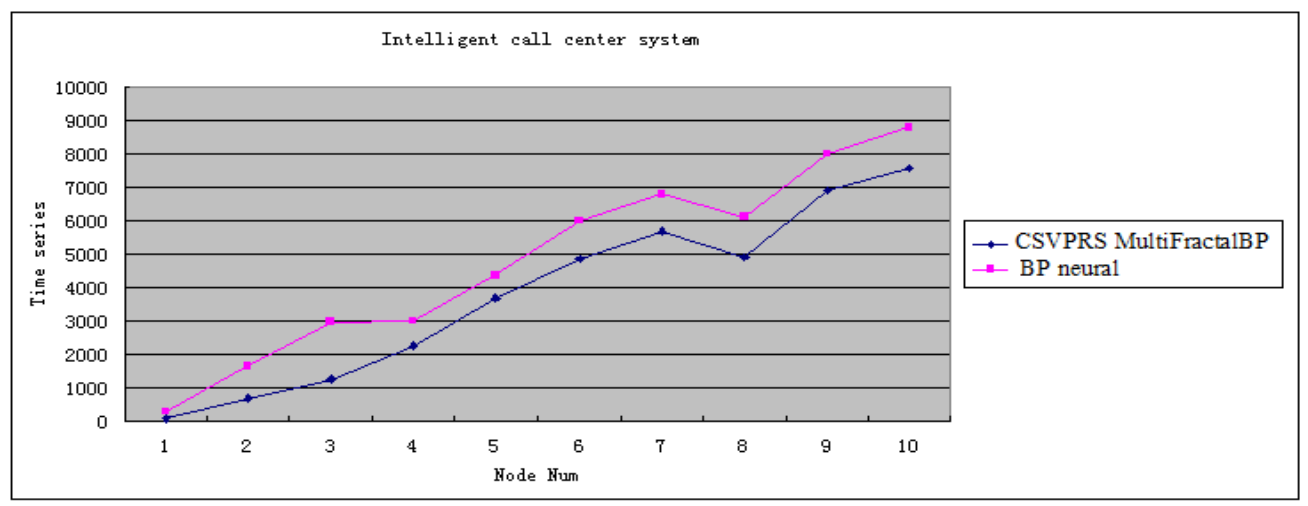

\section{Figure 2. Comparison of Intelligent Call System Based on Cost Sensitive Variable Precision Rough Set and Multi Fractal BP Neural Network Algorithm}

Through the results of Experiment showed that for time series with multifractal properties. The curve is a bell shaped, which is curve under the opening width, said local multi fractal characteristics of distribution, multi fractal dimension of the system is the scope of distribution, including and, these characteristics described together the multifractal properties of the time series on behalf of the system, these features also reflects the time series variation tendency in the future, so as to provide a basis for seating scheduling. In this paper, the customer classification algorithm is improved, the variable precision rough set and BP neural network are used to classify the customers and reduce the influence of missing data and noise data.

\section{Summary}


The paper presents construction of intelligent call center system based on cost sensitive variable precision rough set and multi fractal BP neural network. In intelligent call center business characteristics, project based on rough set theory combined with BP neural network to queue the customer classification and agents using chaotic and fractal theory forecast traffic and optimization of call center scheduling. The system will voice query, self-service, artificial service closely integrated, the shopping website sales, distribution of resources, supply chain resources, customer resources, such as through the network integration, establish a rapid response mechanism of online shopping, enhance customer groups and shopping website interaction, resources to achieve uniform distribution and utilization, so as to achieve the maximum profit for the enterprise.

\section{Acknowledgments}

This paper is supported by the National Natural Science Funds of China (61272015), and also is supported by the science and technology research major project of Henan province Education Department (13B520155) and Henan Province basic and frontier technology research project (142300410303).

\section{References}

[1] C. C. Yeh, D.-J. Chi and M. F. Hsu, "A hybrid approach of DEA, rough set and support vector machines for business failure prediction”, Expert Systems with Applications, vol. 37, no. 2, (2010), pp. 1535-1541.

[2] M. J. Beynon, "An illustration of variable precision rough sets model: an analysis of the endings of the UK Monopolies and Mergers Commission”, Computers \& Operations Research, vol. 32, (2005), pp. 1739-1759.

[3] J. W. Kantelhardt, S. A. Zschiegner and K. Bundle, "Multifractal detrended fluctuation analysis of nonstationary time series", Physical A, vol. 316, no. 14, (2002), pp. 87-114.

[4] J. S. Mi, W. Z. Wu and W. X. Zhang, "Approaches to knowledge reduction based on variable precision rough set model", Information Sciences, vol. 159, (2004), pp. 255-272.

[5] C. W. Bin and C. Jiang, "Forecasting Emergency Demand Based on BP Neural Network and Principal Component Analysis", AISS, vol. 5, no. 13, (2013), pp. 38-45.

[6] J. Lirong, D. Qingli and C. Weida, "Variable Precision Rough Set and a Fuzzy Mea sure of Knowledge Based on Variable Precision Rough Set", Journal of Southeast University (English Edition), vol. 18, no. 4, (2002).

[7] L. Lianxin, L. Yu and S. Guangxia, "Evaluation of quality of teachingbased on BP neural network", Journal of Chemical and Pharmaceutical Research, vol. 6, no. 2, (2014), pp. 83-88.

[8] M. Beynon, "Reduces within the variable precision rough sets model: A further investigation", European Journal of Operational Research, vol. 134, no. 3, (2001), pp. 592-605.

[9] Y. Lv and J. Gao, "Condition prediction of chemical complex systems based on Multifractal and Mahalanobis-Taguchi system", 2011 International Conference on Quality, Reliability, Risk, Maintenance, and Safety Engineering, Xi'an, (2011), pp. 536-539.

[10] M. Beynon, "The Elucidation of an Iterative Procedure to $\beta$ Reduct Selection in the Variable Precision Rough Sets Model”, Berlin: Springer-Verlag, (2004), pp. 412-417.

[11] Y. Lv, J. Gao, Z. Gao and H. Jiang, "Multifractal information fusion based condition diagnosis for process complex", Process Mechanical Engineering, (2012), pp. 1-8.

\section{Authors}

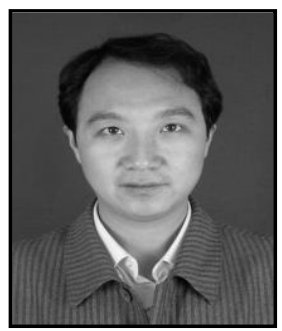

Hongsheng Xu, Birth: Dec 28 ${ }^{\text {th }} 1979$; Educational background: master, Henan University, Kaifeng, China, 2007; Major field of study: Call center, data mining, Knowledge discovery, artificial intelligence, the Semantic Web. 


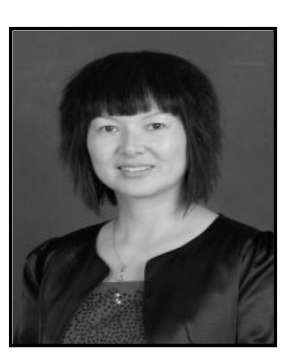

Ruiling Zhang, Birth: Dec $23^{\text {th }}$ 1964; Educational background: master, Northwestern Polytechnical University, Xian, China, 2007; Major field of study: data mining, Knowledge discovery, artificial intelligence, the Semantic Web, Call center.

Chunjie Lin, Birth: Feb 01 ${ }^{\text {th }} 1981$; Educational background: MSE degree in Computer Science from Henan University of Science and Technology, China, 2011; Major field of study: data mining, Call center, Knowledge discovery, artificial intelligence, the Semantic Web. 